\title{
Shallow processing of ambiguous pronouns: Evidence for delay
}

\author{
Andrew J. Stewart, Judith Holler, and Evan Kidd \\ The University of Manchester, Manchester, UK
}

\begin{abstract}
Two self-paced reading-time experiments examined how ambiguous pronouns are interpreted under conditions that encourage shallow processing. In Experiment 1 we show that sentences containing ambiguous pronouns are processed at the same speed as those containing unambiguous pronouns under shallow processing, but more slowly under deep processing. We outline three possible models to account for the shallow processing of ambiguous pronouns. Two involve an initial commitment followed by possible revision, and the other involves a delay in interpretation. In Experiment 2 we provide evidence that supports the delayed model of ambiguous pronoun resolution under shallow processing. We found no evidence to support a processing system that makes an initial commitment to an interpretation of the pronoun when it is encountered. We extend the account of pronoun resolution proposed by Rigalleau, Caplan, and Baudiffier (2004) to include the treatment of ambiguous pronouns under shallow processing.
\end{abstract}

A number of researchers have recently proposed that people do not always arrive at a detailed and coherent interpretation of text as it is read (Ferreira, Bailey, \& Ferraro, 2002; Sanford \& Sturt, 2002). Rather, they claim that readers construct a discourse representation that is "goodenough" for the task at hand. This representation may contain discourse elements that are underspecified. Sanford and Sturt (2002) propose that the depth of processing in which a reader engages influences the level of specification in their resulting discourse representation. Work by Ferreira and colleagues provides empirical evidence for this account, showing that readers sometimes construct underspecified syntactic representations during sentence processing (Christianson, Hollingworth, Halliwell, \& Ferreira, 2001; Ferreira, 2003).

The concept of underspecified representations captures a number of findings in the literature on how readers process anaphora. Levine, Guzman, and Klin (2000) reported several studies that examined how readers interpret noun phrase (NP) anaphora. Participants were presented with passages that contained antecedents (e.g., "tart") followed by NP anaphors (e.g., "dessert"). They found that when the antecedent was

Correspondence should be addressed to Andrew J. Stewart, School of Psychological Sciences, University of Manchester, Oxford Road, Manchester M13 9PL, UK. E-mail: Andrew.Stewart@manchester.ac.uk

We thank Roger van Gompel, Tony Sanford, and two anonymous reviewers for insightful comments on an earlier draft. We thank Matthew Cocksedge for assistance with data collection. This research was funded by British Academy Grant SG-37448 awarded to the first author and was partially supported by a postdoctoral fellowship from the Max Planck Institute for Evolutionary Anthropology awarded to the third author. 
backgrounded, and a same-category distractor (e.g., "cake") was present between anaphor and antecedent, there was no evidence that readers resolved the anaphoric noun phrase (as indexed by participants' reaction times to probe words). Levine et al. (2000) propose that when it is difficult to connect an anaphor to its antecedent, and the text remains sufficiently coherent without that link, readers abandon trying to interpret the anaphor. In other words, readers engage in shallow processing of the anaphor with the result that the referential link between anaphor and antecedent is underspecified (see also Klin, Guzman, Weingartner, \& Ralano, 2006; Klin, Weingartner, Guzman, \& Levine, 2004).

Similar findings have been reported by Greene, McKoon, and Ratcliff (1992), who investigated pronominal anaphora. Using a probe task, they found no evidence for an increase in activation associated with a pronoun's antecedent and proposed a framework in which pronouns are not automatically resolved. In other words, they also found evidence for underspecification of the link between anaphor and antecedent. Importantly, participants in their studies could perform well on the probe task without having fully processed the pronoun. That is, the task allowed them to engage in shallow processing.

In contrast to Greene et al. (1992), the majority of previous work on pronoun resolution has taken for granted that readers do in fact fully specify pronominal referents and thus are typically engaged in deep processing. Much of this work has focused on the time course of the influence of various factors during comprehension (e.g., Arnold, Eisenband, Brown-Schmidt, \& Trueswell, 2000; Garnham, Oakhill, \& Cruttenden, 1992; Stewart, Pickering, \& Sanford, 2000; Sturt, 2003).

A number of papers in the literature have reported studies examining the use of one particular source of information, that of gender, in the resolution of pronominal anaphora (e.g., Arnold et al., 2000; McDonald \& MacWhinney, 1995; Rigalleau \& Caplan, 2000; Rigalleau et al., 2004). Rigalleau et al. (2004) proposed the most complete model of how gender information is used during the interpretation of gender-marked pronouns, which, importantly, admits the possibility that pronouns can be processed at different levels of specification. They propose a two-stage model, similar to an account outlined by Sanford and Garrod (1989). During the first stage, the co-indexation process, gender information is automatically used to check the gender match between the pronoun and the most accessible pronominal referential candidate. During the second stage, processing is more strategic in nature. This stage involves a disengagement process that operates to reduce the activation of the competing noun phrase (i.e., the nonreferent). The authors propose that the first stage operates automatically under all conditions but that this second stage operates only under deep processing, such as when the identity of the pronominal referent is explicitly probed (e.g., through comprehension questions). Rigalleau et al. propose that this model can explain inconsistencies in the literature on the influence of gender information on processing (e.g., Garnham et al., 1992; Rigalleau \& Caplan, 2000). Under shallow processing, where unambiguous pronouns are read in the context of questions that do not probe the referent of the pronoun, the second stage of the model does not operate. In other words, even though the pronoun has been resolved in the sense that the referent has been identified and a link made to it during the co-indexation stage, no mechanism comes into play to reduce the activation of the nonreferent.

In contrast to the co-indexation model, Greene et al. (1992) outlined a model of pronoun resolution under shallow processing whereby they claim that the pronoun is not resolved. This conclusion was made on the basis of a lack of evidence for a difference between the activation levels of pronominal referents and those of nonreferents as measured using a probe task. However, the model detailed by Rigalleau et al. (2004) allows for a reinterpretation of the Greene et al. findings in that the Greene et al. data are consistent with shallow pronominal processing of unambiguous pronouns involving only the co-indexation stage of Rigalleau et al.'s model. As the second stage does not operate, there will be no change to the 
activation level of the nonreferent, thereby explaining Greene et al.'s (1992) null effect. While this model provides a clear framework to account for how gender information is used during the shallow processing of unambiguous pronouns, it does not make any predictions with respect to the shallow processing of ambiguous pronouns. The goal of the experiments reported in the current paper is to examine the shallow processing of ambiguous pronouns and to expand Rigalleau et al.'s (2004) model so that it captures the shallow processing of both unambiguous and ambiguous pronouns.

There are a number of ways in which the shallow processing of ambiguous pronouns may proceed. Consider Sentence 1:

\section{Paul ${ }_{i}$ lent Rick $k_{j}$ the $C D$ before he $e_{i / j}$ left for the holidays.}

The pronoun is ambiguous as it could refer to either character; there is no information available in the sentence to disambiguate reference. In general, there are a limited number of cues available to guide interpretation. These relate to structure, salience of possible referents, and the respective plausibilities of the possible interpretations.

The two-stage model of Rigalleau et al. (2004) can be generalized to include the case of ambiguous pronouns under shallow processing. With unambiguous pronouns, the first stage of the model involves automatic co-indexation between pronoun and gender-consistent referent, as in Sentence 2:

\section{Paul $l_{i}$ lent Claire $j_{j} \mathrm{CD}$ before she $\mathrm{j}_{\mathrm{j}}$ left for the holidays.}

When the pronoun is encountered, this coindexation stage operates. It may then be followed by the second, more strategic, stage whereby the activation of the nonreferent, Paul, is reduced. However, in the case of Example 1, gender information cannot be used to drive an initial coindexation stage. In this situation, if co-indexation occurs, it must be governed by another source of information. One possibility is that the co-indexation stage is driven by a heuristic resulting in (for example) co-indexation between the pronoun and the first-mentioned character. Sentential subjects are often argued to hold privileged status because they are frequently topicalized, and there is considerable evidence that the sentential subject, also the first-mentioned character in this example, occupies a privileged position within a reader's discourse model (Gernsbacher \& Hargreaves, 1988). Such a heuristic would be useful in the case of Sentence 1.

A second possibility is that there is no coindexation of ambiguous pronouns when they are encountered under shallow-processing conditions. This predicts that, as there is no need to interpret the ambiguous pronouns in the context of shallow processing (i.e., where the referent of the pronoun does not have to be identified), and as there is no information to guide interpretation anyway, they simply go unresolved. Both of these accounts predict that reading times should be equivalent for ambiguous and unambiguous pronouns under shallow processing (although for different reasons). For unambiguous pronouns, co-indexation will rapidly and automatically be made between pronoun and referent on the basis of gender information. For ambiguous pronouns, if co-indexation occurs, this will be made on the basis of a heuristic such as co-indexing the pronoun and the first-mentioned character. This will proceed in a rapid manner. Alternatively, if co-indexation does not occur then no processing cost will accrue.

Under deep processing, both of these models predict that reading times for ambiguous pronouns will be longer than those for unambiguous pronouns. This is because the second stage of Rigalleau et al.'s (2004) model involves a disengagement process that results in a strategic suppression of the activation of the nonreferent. For sentences where no information is available to clearly confirm or deny the first-mentioned character as being the referent, and the plausibilities of the two possible interpretations are similar, an increase in reading times would be observed as the reader will have to engage strategically in order to deselect the nonreferent.

Alternatively, readers may always attempt to quickly arrive at the most likely interpretation of an ambiguous pronoun regardless of depth of 
processing (perhaps because it is cognitively costly to delay interpretation). If that is the case, we would expect that sentences containing ambiguous pronouns will always take longer to read regardless of whether processing is shallow or deep.

\section{EXPERIMENT 1}

\section{Plausibility pretest}

In order to ensure that readers could not simply use plausibility information to guide interpretation of the pronoun, a plausibility pretest was conducted to ensure that both interpretations of the test sentences were equally available to adult speakers. Plausibility may be a strong cue available to guide interpretation under normal circumstances. However, for the purposes of our investigation it is important to be able to rule out plausibility differences as an explanation for any findings we report. Although this may limit the generalizability of our findings, without being able to discount plausibility we would be unable to propose any model that did not feature plausibility in a central role. A total of 24 sentences were used; two versions were created of each. One version contained an unambiguous pronoun referring to the mainclause grammatical subject (e.g., Paul lent Kate the CD before he left for the holidays; the "first character reference" condition); the other version contained an unambiguous pronoun referring to the main-clause grammatical object (e.g., Paul lent Kate the CD before she left for the holidays; the "second character reference" condition). Two versions of the questionnaire were generated, each containing either the first character reference sentence or the second character reference sentence of any given test sentence. Each questionnaire contained equal amounts of first and second character reference sentences. The questionnaires were given to two independent groups of 10 native English speakers who were asked to rate each sentence for plausibility of the event it described on a 7-point Likert scale (with 1 on the scale corresponding to "highly implausible" and 7 to "highly plausible"). The average plausibility of the versions referring the first-mentioned character was 5.75, and the average plausibility of the versions referring to the second-mentioned character was 5.80. For each pair, the difference between the two versions was $\leq 0.75$. Analysis of variance revealed no difference in the plausibilities between the versions that referred to the first-mentioned character and the versions that referred to the second (both $F_{\mathrm{S}}<1$ ).

\section{Method}

\section{Participants}

A total of 54 participants took part in the experiment, drawn from the University of Manchester population. All participants were native English speakers. They did not have a reading disability and were not involved in any aspects of linguistic or psycholinguistic studies. No participants had taken part in the pretest.

\section{Design and stimuli}

The experiment employed a $2 \times 3$ mixed design, consisting of a between-subjects factor, "depth of processing", with two levels ("shallow processing" vs. "deep processing”), and a within-subjects factor, "ambiguity", with three levels (ambiguous, first character reference, second character reference). The stimulus material consisted of 24 target sentences and 20 filler sentences. Three versions of each sentence were generated:

1. Ambiguous (Paul lent Rick the CD before he left for the holidays).

2. First character reference (Paul lent Kate the CD before he left for the holidays).

3. Second character reference (Paul lent Kate the CD before she left for the holidays).

Three different lists were created from these sentences using the Latin Square technique. Each list contained eight sentences of each of the three different types. The same 20 filler sentences were added to each list. The filler items were of the same general format (i.e., two characters engaged in some activity). Each filler also contained an anaphor referring to one of the two characters. These anaphors were a mixture of globally ambiguous pronouns, unambiguous pronouns, and unambiguous repeated names. 
For our "shallow-processing" group, questions requiring a yes/no answer (e.g., Did Paul lend Kate the CD?/Did Kate lend Paul the CD?, respectively) were paired with a third of the target sentences and a third of the filler items. Overall, half of the questions probed the information content of the main clause and half the information content of the subordinate clause. The other two thirds of the experimental and filler items had the phrase "No question" following them. For our deep-processing group, questions on both experimental items and fillers probed the information content of the subordinate clause and so required resolution of the pronoun (e.g., "Who lent the CD? Rick or Paul"). All experimental and filler sentences were followed by a question in the deep-processing conditions.

\section{Procedure}

Participants were tested individually and were randomly allocated to one of the two experimental groups. Each participant was provided with verbal as well as standardized written instructions. These informed them that a cross would appear at the left-hand side of the computer screen, followed by a short sentence. For the shallow-processing group, participants were instructed to read the sentence and then to press the middle button on a button box. They would then see a yes/no question relating to the preceding sentence (or the words "No question", in which case they should simply press the middle button to move on to the next sentence). They were told that this question should be answered as quickly and as accurately as possible, using the buttons marked "Yes" and "No" on the button box. For the deepprocessing group, the procedure employed was identical except that when the question was displayed on the computer screen it was followed by the two character names that had appeared in the preceding sentence (in half of the cases the names were in the same order as they appeared in the sentence, and in half of the cases the order was reversed-participants were told at the beginning that the names did not necessarily match the order in which they occurred in the sentence). Participants had to press the left button when they believed the answer to be the name displayed in left-hand position on the screen and the right button when they thought that the answer was the name displayed in the right-hand position on the screen. The next trial was then presented.

Participants in both processing conditions completed eight practice trials before beginning the actual experiment. Half of the practice trials contained globally ambiguous pronouns (e.g., Larry ran after Mick because he forgot his wallet) while the other half contained pronouns and were unambiguous because of a gender contrast between the two characters in the main clause. Practice trials in the deep-processing condition all had questions that could only be correctly answered if participants had resolved the referent of the pronoun in the practice items (e.g., Who forgot his wallet? Larry or Mick). For the shallow-processing condition only a third of the practice trials were paired with a question, and those questions could be answered without the participants having resolved the referent of the pronoun (e.g., Did Larry run after Mick?). The motivation behind the practice trials was twofold. We wanted participants to be comfortable with the experimental set-up and clear on what buttons should be pressed during reading of the materials and answering of the questions. More importantly, we wanted to try and put the participants in each processing condition in a mode of reading that encouraged them to construct either a fully specified or an underspecified discourse model before the experiment itself began. At the end of the experiment each participant was fully debriefed, thanked, and financially compensated.

The experiment was run using the E-prime programming software (MacWhinney, St James, Schunn, Li, \& Schneider, 2001). A button box recorded participants' reading times. The order in which the sentences were presented was randomized by the computer.

\section{Analysis}

The data were analysed for the total time (in milliseconds) participants took to read each of the three sentence types (ambiguous vs. first character 
reference vs. second character reference) for both levels of processing (shallow vs. deep) and for the time participants took to answer the questions relating to the different sentence types under deep-processing and shallow-processing conditions. Another part of the analysis evaluated the percentage of questions correctly answered under deep-processing processing for each of the first character reference and the second character reference sentences. For the ambiguous sentences, we evaluated how often participants resolved the ambiguity by interpreting the ambiguous pronoun as referring to the first-mentioned or the second-mentioned character.

\section{Results}

\section{Sentence reading-time data}

We conducted a 3 (ambiguity: ambiguous vs. first character reference vs. second character reference) $\times 2$ (processing: shallow processing vs. deep processing) analysis of variance (ANOVA) on the sentence reading-time data. This revealed a main effect of ambiguity, $F_{1}(2,104)=3.475, M S E=$ 406,961, $p<.05 ; F_{2}(2,92)=4.502, M S E=$ $500,481, p<.05$, no effect of processing (both $F_{\mathrm{S}}<1$ ), and an interaction between ambiguity and processing, $F_{1}(1,104)=5.321, M S E=$ 406,961, $p<.01 ; F_{2}(2,92)=3.305, M S E=$ 500,481, $p<.05$. (See Table 1.)

In the shallow-processing condition, planned comparisons revealed that none of the conditions differed from each other (all $F_{\mathrm{S}}<1$ ). In the deep-processing condition, the ambiguous

Table 1. Experiment 1: Sentence reading times

\begin{tabular}{llllll}
\hline & \multicolumn{4}{c}{ Processing } \\
\cline { 2 - 5 } & \multicolumn{2}{c}{ Deep } & & \multicolumn{2}{c}{ Shallow } \\
\cline { 2 - 3 } \cline { 5 - 6 } & Mean & SE & & Mean & SE \\
\hline Ambiguous & 4,714 & 236 & 4,183 & 261 \\
First character reference & 4,047 & 152 & 4,254 & 249 \\
Second character reference & 4,136 & 147 & 4,273 & 224 \\
\hline
\end{tabular}

Note: Reading times in ms. condition was read more slowly than both the first character reference condition, $F_{1}(1,26)=$ 10.135, $M S E=1,185,085, p<.005 ; F_{2}(1,23)$ $=15.697, M S E=697,136, p<.005$, and the second character reference condition, $F_{1}(1,26)=$ 8.641, $M S E=1,043,037, p<.01 ; F_{2}(1,23)=$ 14.584, $M S E=524,990, p<.005$. There was no difference between the first character reference and second character reference conditions (both $\left.F_{\mathrm{S}}<1\right)$.

\section{Question response time analysis}

For the deep-processing condition, we conducted a one-way ANOVA (ambiguous vs. first character reference vs. second character reference) on the question reaction time data. This revealed a main effect of condition, $F_{1}(2,52)=19.74, M S E=$ $105,196, p<.001 ; F_{2}(2,46)=10.29, M S E=$ 99,659, $p<.001$. Question answering times were significantly slower in the ambiguous condition than in both the first character reference condition, $F_{1}(1,26)=22.520, M S E=251,412$, $p<.001 ; F_{2}(1,23)=13.184, M S E=117,473$, $p<.005$, and the second character reference condition, $F_{1}(1,26)=32.172, M S E=209,786, p<$ $.001 ; F_{2}(1,23)=13.020, M S E=309,093, p<$ .005 . There was no difference between the first character reference and second character reference conditions (both $F_{\mathrm{S}}<1$ ). For the shallow-processing condition, as questions appeared on only a third of the items, and that was not rotated across lists, we report by-items analyses only. We conducted a one-way ANOVA (ambiguous vs. first character reference vs. second character reference) on the question reaction time data. This revealed no effect of condition, $F_{2}(2,14)=$ 2.005, $M S E=122,374, p=.171$ ). (See Table 2.)

\section{Question response accuracy analysis}

For the deep-processing condition, participants responded at $88 \%$ accuracy for questions following first character reference sentences and at 92\% accuracy for questions following second character reference sentences. This difference was not significant, $F_{1}(1,26)=2.608, M S E=0.007, p=$ $.118 ; F_{2}(1,23)=1.108, M S E=0.015, p=$ .303. Overall, for the ambiguous sentences 
Table 2. Experiment 1: Question answering reaction times

\begin{tabular}{lrrrrr}
\hline & \multicolumn{4}{c}{ Processing } \\
\cline { 2 - 3 } & \multicolumn{2}{c}{ Deep } & & \multicolumn{2}{c}{ Shallow } \\
\cline { 2 - 3 } \cline { 5 - 6 } & Mean & SE & Mean & $S E$ \\
\hline Ambiguous & 2,197 & 119 & 2,816 & 166 \\
First character reference & 1,739 & 99 & 2,696 & 151 \\
Second character reference & 1,697 & 88 & 3,041 & 162 \\
\hline
\end{tabular}

Note: Reaction times in ms.

participants responded by pressing the button corresponding to the first character $54 \%$ of the time and the button corresponding to the second character $46 \%$ of the time. This difference between answers that selected the first-mentioned character and answers that selected the second-mentioned character for questions following ambiguous sentences was not statistically significant $(p=.839)$.

For the shallow-processing condition, participants responded at $92 \%$ accuracy for questions following first character reference sentences and at 93\% accuracy for questions following second character reference sentences. Following ambiguous sentences, participants responded at $76 \%$ accuracy. This difference between the conditions was not significant, $F_{2}(2,14)=2.956, M S E=2.3, p=$ .085). (See Table 3.)

\section{Discussion}

Our manipulation of processing depth had a strong impact on the pattern of effects that we

Table 3. Experiment 1: Question response accuracy

\begin{tabular}{llllll}
\hline & \multicolumn{4}{c}{ Processing } \\
\cline { 2 - 4 } & \multicolumn{2}{c}{ Deep } & & \multicolumn{2}{c}{ Shallow } \\
\cline { 2 - 3 } \cline { 5 - 6 } & Mean & SE & & Mean & SE \\
\hline Ambiguous & - & & 76.4 & 9.7 \\
First character reference & 88.4 & 2.1 & 91.7 & 3.4 \\
Second character reference & 92.1 & & 2.2 & 93.1 & 2.0 \\
\hline
\end{tabular}

Note: Accuracy in percentages. found. When participants were required to engage in deep processing, the data support the proposal that readers invest processing resources to interpret the ambiguous pronoun, as indicated by greater reading times for the sentences containing the ambiguous pronouns than for those containing the unambiguous pronouns in the deep-processing condition. Conversely, under shallow processing there was no evidence of processing difficulty associated with reading sentences containing ambiguous pronouns. There is some evidence in the question accuracy data, however, to suggest that the semantic representations that are constructed following sentences containing ambiguous pronouns may not be as stable as those constructed following sentences containing unambiguous pronouns as participants answered fewer comprehension questions correctly in the ambiguous condition.

Our finding in the Experiment 1 data that the two unambiguous versions of our sentences took the same length of time to read as the ambiguous version under shallow processing is consistent with a generalized version of Rigalleau et al.'s (2004) model. There are two possible versions of a generalized model that we consider below. For ambiguous pronouns, one version of the model allows for coindexation via a heuristic. A second generalized version does not allow for co-indexation. However, it is not clear following Experiment 1 which of these possible accounts is correct. In the Introduction we outlined a heuristic that preferentially co-indexes the pronoun and first-mentioned character, but there are possible alternative heuristics, which we outline below. Following Experiment 1, all we can be sure of is that shallow processing of ambiguous pronouns is different from deep processing. In Experiment 2 we further explore what happens to ambiguous pronouns during shallow processing and contrast three possible accounts based on the Rigalleau et al. model.

\section{EXPERIMENT 2}

Several possible variations of the Rigalleau et al. model follow from the Experiment 1 data. Each 
makes a different set of predictions under conditions where disambiguating information is provided to the reader downstream from the ambiguity. In Experiment 2 we achieve this disambiguation by including a second sentence in which a disambiguating region allows the reader to unambiguously determine the reference of the ambiguous pronoun. By examining reading times to the disambiguating region, it is possible to establish how the reader analysed the ambiguous pronoun in the first sentence under shallow processing.

In Experiment 2 we focus only on shallow processing and present participants with two-sentence materials (see Examples 4-7).

4. Paul lent Rick the CD before he left for the holidays. He went to the Bahamas and sent Rick a postcard from the hotel. (Ambiguous, first character reference condition.)

5. Paul lent Rick the CD before he left for the holidays. He went to the Bahamas and sent Paul a postcard from the hotel. (Ambiguous, second character reference condition.)

6. Paul lent Kate the CD before he left for the holidays. He went to the Bahamas and sent Kate a postcard from the hotel. (Unambiguous, first character reference condition.)

7. Kate lent Rick the CD before he left for the holidays. He went to the Bahamas and sent Kate a postcard from the hotel. (Unambiguous, second character reference condition.)

Sentence 1 in each pair is the same as our sentences in Experiment 1. Sentence 2 contains a pronoun. This pronoun will be interpreted as referring to the same character as the pronoun in Sentence 1 . Sentence 2 also contains a disambiguating region whereby one possible referent is ruled out. This disambiguation occurs at the point of the occurrence of the repeated name of the character to which the pronoun does not refer.

In Experiment 2 we use a word-by-word selfpaced reading paradigm to test the competing predictions of three models. The first two models that we discuss predict co-indexation when the ambiguous pronoun is first encountered. This might then be revised should disambiguating information suggest that revision is necessary. Within the context of a generalized version of Rigalleau et al.'s model, this revision stage is equivalent to the disengagement stage.

The first of these "initial commitment" models is the first-mentioned character preference account.
This is the account that we mentioned in our Introduction. It predicts that readers will coindex the ambiguous pronoun in Sentence 1 with the first-mentioned character when it is encountered in Sentence 1 (Crawley, Stevenson, \& Kleinman, 1990; Gernsbacher \& Hargreaves, 1988). If this is the case, then following an ambiguous pronoun we expect to find evidence that it takes longer to read the disambiguating region in Sentence 2 when it disambiguates towards the second-mentioned character than when it disambiguates towards the first-mentioned character. During the second stage of a generalized version of Rigalleau et al.'s model, revision will be necessary to allow for the pronoun to be interpreted as coreferential with the second-mentioned character. With respect to the unambiguous pronouns, this position also suggests that we should find evidence that it is easier to refer to the firstmentioned character than it is to refer to the second-mentioned character. This effect would be expected to arise on the pronoun region in Sentence 1 and on the pronoun region in Sentence 2.

The second of these "initial commitment" models is the unrestricted race model. This type of model has recently been put forward to account for the processing of sentences containing global syntactic ambiguities (van Gompel, Pickering, \& Traxler, 2000, 2001). The model proposes that possible analyses of a global syntactic ambiguity are engaged in a "race" and that the analysis that is constructed fastest is the one that ends up being adopted. A similar mechanism could be proposed to account for the processing of ambiguous pronouns. It would predict that during the co-indexation stage participants will sometimes co-index the pronoun in Sentence 1 with the first-mentioned character and sometimes with the second-mentioned character. Roughly half of the time this co-indexation will turn out to be correct (and half of the time incorrect). Revision will therefore be necessary (roughly) half of the time. Such revision would occur during the second stage of a generalized version of Rigalleau et al.'s model. This will result in an elevation of reading times to the disambiguating region in 
Sentence 2 in our ambiguous conditions as half of the time the reader will have to reinterpret the pronoun following an incorrect co-indexation.

In line with effects that have been reported studying syntactic ambiguity, the unrestricted race model predicts that the region containing the pronoun in Sentence 1 will be read more quickly in the ambiguous conditions than in the unambiguous conditions. The model predicts that whatever interpretation of the pronoun it is that wins the "race" is adopted at this point. The unrestricted race model also predicts a difference between the ambiguous and unambiguous conditions at the disambiguating region in Sentence 2 (i.e., the point at which the repeated name occurs). It predicts that this region will show evidence of an elevation in reading time in the ambiguous conditions compared to the unambiguous conditions. For the ambiguous conditions, half of the time the co-indexation that won the race when the pronoun was encountered in Sentence 1 will turn out to be incorrect, and so the reader will have to reinterpret the pronoun at this point (thus leading to elevated reading times).

The third model is the delayed assignment account. In contrast to the two "initial commitment" accounts, it does not propose that processing of the ambiguous pronoun results in initial co-indexation. Rather, it predicts a delay in processing (where this processing is equivalent to the operation of the second, strategic, stage of Rigalleau et al.'s model) and that this delay persists until disambiguating information is available. Both Garnham et al. (1992) and Rigalleau et al. have shown that interpreting an unambiguous pronoun under shallow processing does not lead to a processing cost. This is consistent with what we reported for the shallow-processing conditions in Experiment 1. Rigalleau et al.'s original model proposes that the gender co-indexation stage of pronoun resolution is an automatic process that operates regardless of whether the reader is engaged in shallow or deep processing. On the basis of our Experiment 1 data and on data reported in Garnham et al. (1992), it also appears to be a fast-acting process. Therefore, the delayed assignment model predicts no difference between the ambiguous and unambiguous conditions in terms of reading times to the region containing the pronoun in Sentence 1. For the ambiguous conditions, it predicts that assignment will be delayed until disambiguating information is available to guide interpretation. For the unambiguous conditions, it predicts that assignment is trivial and results from automatic co-indexation. This means that reading times for the pronoun region in Sentence 1 will be expected to be equivalent across conditions.

The delayed assignment model predicts an effect on the disambiguating region in Sentence 2 in the ambiguous conditions. This will be an increase in reading time in the ambiguous conditions, as this is when the reader encounters the information necessary to allow for clear interpretation of the pronoun. Only when they encounter this information can they strategically select the pronominal reference (and deselect the nonreferent). Because this account does not involve an initial co-indexation between an ambiguous pronoun and referent (followed by possible revision), it does not predict any difference between the disambiguating region when it disambiguates towards the first-mentioned character and the same region when it disambiguates towards the second.

\section{Method}

\section{Participants}

A total of 44 participants took part in this experiment, fulfilling the same criteria as did those who took part in Experiment 1.

\section{Design and stimuli}

A $2 \times 2$ design was used with the first factor being ambiguity (ambiguous vs. unambiguous) and the second factor character (first character reference vs. second character reference). The materials consisted of two sentences. Comprehension questions were of the shallow-processing kind and on a third of the trials and fillers. Overall, the questions probed the information content in the main clause of Sentence 1 half the time and content in the subordinate clause of Sentence 1 half the 
time. The stimulus material for Experiment 2 consisted of 24 two-sentence materials and 20 twosentence fillers. The reading times for each word in Sentences 1 and 2 were recorded. For each pair of sentences, Sentence 1 was the same as the target sentences used in Experiment 1. When following an unambiguous Sentence 1, Sentence 2 was always referentially congruent with Sentence 1. When following an ambiguous Sentence 1, Sentence 2 disambiguated towards the first-mentioned character in Sentence 1 a total of $50 \%$ of the time and the second-mentioned character in Sentence 1 a total of $50 \%$ of the time.

\section{Procedure}

The pairs of sentences were presented word by word in a cumulative fashion. We presented the sentence cumulatively so that participants could make regressions to the first sentence when they were resolving the pronoun in the second sentence. At the start of each trial a line of dashes appeared on the screen corresponding to word boundaries. Participants then pressed the middle button on the button box for the first word to appear. Once they had read each word they were instructed to press the middle button again to reveal the next word (and so on). At the end of each trial participants then had to press another button on the button box. They would then be presented with a yes/no question relating to the preceding sentence (or with the phrase "no question", in which case they were told simply to press the middle button to move on to the next sentence). They were told that this question should be answered as quickly and as accurately as possible, using the buttons marked "Yes" and "No" on the button box. The next trial was then presented.

\section{Analysis}

The data were analysed for the total time (in milliseconds) that participants took to read each of three regions in Sentence 1 and each of four regions in Sentence 2 for the three sentence types (ambiguous, first character reference, second character reference). In Example 7, dashes correspond to region splits. The question response accuracy and question response reaction time data were also analysed.

7. Paul lent Rick the CD before/he left/for the holidays./He went/to the Bahamas and sent/Rick a/postcard from the hotel.

Two of the regions are critical in that they are the regions for which the models make different predictions. These are the pronoun region in Sentence 1 and the disambiguating region in Sentence 2. In light of previous work (e.g., Ehrlich \& Rayner, 1983) that has shown that effects related to the processing of a pronoun often occur on the word following the pronoun, we defined each of these regions as the anaphoric word (i.e., pronoun or repeated name) plus the following word.

\section{Results}

We conducted a two (ambiguous vs. unambiguous) by two (first character reference vs. second character reference) ANOVA on each region, on the question reaction times and on the question response accuracy.

\section{Region before the pronoun in Sentence 1}

On this region we found no effect of ambiguity, $F_{1}(1,43)=1.377, M S E=401,608, p=.247$; $F_{2}(1,23)=1.982, M S E=152,236, p=.173$, no effect of character, $F_{1}(1,43)=3.367, M S E$ $=383,133, p=.073 ; F_{2}(1,23)=3.781, M S E=$ 186,087, $p=.064$, and no interaction (both $F_{\mathrm{S}}$ $<$ 1). (See Table 4.)

\section{Pronoun region in Sentence 1}

On this region we found no effect of ambiguity (both $F_{\mathrm{S}}<1$ ), no effect of character, $F_{1}(1,43)$ $=3.089, M S E=31,077, p=.086 ; F_{2}(1,23)=$ $1.265, M S E=41,402, p=.272$, and no interaction (both $F_{\mathrm{s}}<1$ ).

\section{Region after the pronoun in Sentence 1}

On this region we found no effect of ambiguity (both $F_{\mathrm{S}}<1$ ), no effect of character (both $F_{\mathrm{S}}<$ 1 ), and no interaction (both $F_{\mathrm{S}}<1$ ). 
Table 4. Experiment 2: Reading times within each region for Sentence 1

\begin{tabular}{|c|c|c|c|c|c|c|c|}
\hline & \multirow[b]{3}{*}{ Character } & \multicolumn{6}{|c|}{ Region } \\
\hline & & \multicolumn{2}{|c|}{ Before pronoun } & \multicolumn{2}{|c|}{ Pronoun } & \multicolumn{2}{|c|}{ After pronoun } \\
\hline & & Mean & $S E$ & Mean & $S E$ & Mean & $S E$ \\
\hline \multirow[t]{2}{*}{ Ambiguous } & First & 2,563 & 185 & 736 & 59 & 1,393 & 158 \\
\hline & Second & 2,389 & 152 & 674 & 35 & 1,392 & 146 \\
\hline \multirow[t]{2}{*}{ Unambiguous } & First & 2,448 & 131 & 699 & 43 & 1,420 & 111 \\
\hline & Second & 2,280 & 130 & 668 & 49 & 1,338 & 118 \\
\hline
\end{tabular}

Note: Reading times in ms.

\section{Pronoun region in Sentence 2}

On this region we found no effect of ambiguity (both $F_{\mathrm{S}}<1$ ), no effect of character (both $F_{\mathrm{S}}<$ $1)$, and no interaction, $F_{1}(1,43)=1.592, M S E$ $=99,097, p=.214 ; F_{2}(1,23)=2.670, M S E=$ 42,342, $p=.116$.) (See Table 5.)

\section{Region after the pronoun in Sentence 2}

On this region we found no effect of ambiguity (both $F_{\mathrm{S}}<1$ ), no effect of character (both $F_{\mathrm{S}}<$ $1)$, and no interaction, $F_{1}<1 ; F_{2}(1,23)=$ $1.141, M S E=152,481, p=.297$.

\section{Disambiguating region in Sentence 2}

On this region we found an effect of ambiguity, $F_{1}(1,43)=5.468, M S E=217,303, p=.024$; $F_{2}(1,23)=11.072, M S E=48,889, p=.003$, with this region being read more slowly in the ambiguous conditions $(1,062 \mathrm{~ms})$ than in the unambiguous conditions $(897 \mathrm{~ms})$. There was no effect of character, $F_{1}<1 ; F_{2}(1,23)=1.035$, $M S E=92,962, p=.320$, and no interaction (both $F_{\mathrm{S}}<1$ ).

\section{Region after the disambiguating region in Sentence 2}

On this region we found no effect of ambiguity, $F_{1}(1,43)=1.589, M S E=581,049, p=.214$; $F_{2}(1,23)=1.555, M S E=253,141, p=.226$, no effect of character (both $F_{\mathrm{S}}<1$ ), and no interaction (both $F_{\mathrm{S}}<1$ ).

\section{Question response accuracy}

We found no effect of ambiguity, $F_{1}(1,43)=$ 3.716, $M S E=0.075, p=.061 ; F_{2}(1,7)=$ $1.960, M S E=0.026, p=.204,{ }^{1}$ an effect of character that was significant by participants only, $F_{1}(1,43)=6.510, M S E=0.071, p=.014$; $F_{2}(1,7)=2.224, M S E=0.038, p=.180$, and no interaction (both $F_{\mathrm{S}}<1$ ). (See Table 6.)

\section{Question response times}

We found no effect of ambiguity, $F_{1}<1 ; F_{2}(1,7)$ $=1.096, M S E=459,257, p=.330$, no effect of character (both $F_{\mathrm{S}}<1$ ), and no interaction, $F_{1}(1,43)=1.314, M S E=508,203, p=.258$; $F_{2}<1$.

\section{Discussion}

We can rule out the first-mentioned character preference account as our analyses of the disambiguation region showed that it was no more difficult to recover from disambiguation towards the secondmentioned character than it was to recover from disambiguation towards the first. Indeed, the trend for the disambiguating region actually goes in the opposite direction from that predicted by the first-mentioned character preference account.

\footnotetext{
${ }^{1}$ As with Experiment 1 there is a numerical (but nonstatistically significant) difference in comprehension accuracy between the ambiguous and unambiguous conditions.
} 
Table 5. Experiment 2: Reading times within each region for Sentence 2

\begin{tabular}{|c|c|c|c|c|c|c|c|c|c|}
\hline & \multirow[b]{3}{*}{ Character } & \multicolumn{8}{|c|}{ Region } \\
\hline & & \multicolumn{2}{|c|}{ Pronoun } & \multicolumn{2}{|c|}{ After pronoun } & \multicolumn{2}{|c|}{ Disambiguating } & \multicolumn{2}{|c|}{$\begin{array}{c}\text { After } \\
\text { disambiguating } \\
\text { region }\end{array}$} \\
\hline & & Mean & $S E$ & Mean & $S E$ & Mean & $S E$ & Mean & $S E$ \\
\hline \multirow[t]{2}{*}{ Ambiguous } & First & 891 & 75 & 1,717 & 131 & 1,099 & 112 & 2,580 & 202 \\
\hline & Second & 806 & 62 & 1,778 & 162 & 1,024 & 98 & 2,510 & 184 \\
\hline \multirow[t]{2}{*}{ Unambiguous } & First & 817 & 85 & 1,818 & 117 & 909 & 83 & 2,432 & 200 \\
\hline & Second & 853 & 80 & 1,710 & 115 & 885 & 73 & 2,369 & 194 \\
\hline
\end{tabular}

Note: Reading times in ms.

There was no statistically robust evidence in either the pronoun region in Sentence 1 or the pronoun region in Sentence 2 that unambiguous pronouns were read more quickly when they referred to the first- rather than the second-mentioned character.

Contrary to the unrestricted race account we found no evidence in our analyses of the pronoun region in Sentence 1 that globally ambiguous pronouns were read more quickly than unambiguous pronouns. Overall, our data fit best with the predictions of the delayed assignment account. We found no evidence of initial co-indexation of ambiguous pronouns, but strong evidence in our analyses of the disambiguation region in Sentence 2 that readers were delaying interpretation of the ambiguous pronouns until disambiguating information was available. In other words, referents of ambiguous pronouns remained underspecified until disambiguating information was

Table 6. Experiment 2: Question response accuracy and question reaction times

\begin{tabular}{|c|c|c|c|c|c|}
\hline & \multirow[b]{2}{*}{ Character } & \multicolumn{2}{|c|}{$\begin{array}{l}\text { Question } \\
\text { response } \\
\text { accuracy }^{\mathrm{a}}\end{array}$} & \multicolumn{2}{|c|}{$\begin{array}{l}\text { Question } \\
\text { reaction time }\end{array}$} \\
\hline & & Mean & $S E$ & Mean & $S E$ \\
\hline \multirow[t]{2}{*}{ Ambiguous } & First & 75.0 & 5.3 & 1,741 & 124 \\
\hline & Second & 69.3 & 4.9 & 1,891 & 164 \\
\hline \multirow[t]{2}{*}{ Unambiguous } & First & 87.5 & 3.3 & 1,756 & 111 \\
\hline & Second & 72.7 & 5.3 & 1,660 & 129 \\
\hline
\end{tabular}

${ }^{\mathrm{a}}$ In percentages. ${ }^{\mathrm{b}} \mathrm{In} \mathrm{ms}$. encountered, and the second, strategic, stage of Rigalleau et al.'s model operated.

\section{GENERAL DISCUSSION}

In two experiments we explored how the processing of ambiguous pronouns occurs under shallow processing. We have shown that Greene et al.'s (1992) proposal that pronoun resolution does not occur under shallow processing is incorrect. Rather we found evidence of an initial underspecification of ambiguous pronouns that is the result of an absence of co-indexation (in contrast to what Rigalleau et al. (2004) reported for unambiguous pronouns). This initial underspecification then becomes more fully specified when disambiguating information is encountered. We found no evidence that readers were making an initial commitment that was open to later revision. The data associated with the shallow processing of ambiguous pronouns are consistent with the sole operation of the second stage of the two-stage model proposed by Rigalleau et al. The evidence for a delay in interpretation of ambiguous pronouns means that the first stage of a more general version of this model does not produce an initial co-indexation in the case of shallow processing of ambiguous pronouns. Interpretation only takes place when information to clearly guide that interpretation becomes available. The most likely explanation then is that readers engaged in 
strategic processing of ambiguous pronouns at the point at which disambiguation was encountered. This finding that strategic processing of ambiguous pronouns does take place under shallow processing (albeit after a delay) is in contrast to the position for unambiguous pronouns described by Rigalleau et al. A more generalized version of this model must allow for this difference in the shallow processing of ambiguous and unambiguous pronouns. One possible reason as to why strategic, delayed processing of ambiguous pronouns occurred might be because of the need to maintain referential coherence across utterances. In the case of unambiguous pronouns, co-indexation itself results in referential coherence without the need for any strategic input. Consistent with Rigalleau et al.'s model (and with data reported by Garnham et al., 1992), we found that the shallow processing of unambiguous pronouns occurs without any measurable processing cost.

In Experiment 1 we found that ambiguous pronouns are processed differently depending on whether they occur in contexts of shallow or deep processing. In Experiment 2 we tested the predictions of three models to examine how readers were processing the ambiguous pronouns under shallow processing. We presented a second sentence, which disambiguated reference towards the first character $50 \%$ of the time following an ambiguous first sentence. If readers did not resolve the ambiguous pronouns at any point, then we would have found no evidence of an elevation for any region in the second-sentence reading times corresponding to what we argue is the delayed instantiation of the link between ambiguous pronoun and antecedent.

Although previous work has also shown that ambiguous pronouns may not always be fully interpreted as soon as they are encountered, our data show that an important influencing factor on interpretation is the depth of processing in which a reader is engaged. In other words, it is not the case that resolution of ambiguous pronouns is usually delayed per se but, rather, whether or not it is delayed is determined by depth of processing engaged in by participants in experimental situations. If comprehension questions require resolution to take place, then readers will assign a referent to the ambiguous pronoun even in a context where no disambiguation is available to indicate which assignment is correct. Under shallow processing, ambiguous pronouns will ultimately be interpreted but only when the reader is able to do this with certainty. This suggests that pronominal anaphors are treated differently from noun phrase anaphors of the type studied by Levine et al. (2000). They found no evidence that under shallow processing these anaphors were ever fully resolved. We found that resolution of ambiguous pronominal anaphors does take place when disambiguating information becomes available.

In contrast to the findings of Greene et al. (1992), our data show that pronoun resolution is delayed rather than nonexistent in the case of ambiguous pronouns under shallow processing. In the materials of Greene et al. (1992), the pronoun always appeared in the last sentence of their passages. Our findings show that, while resolution of an ambiguous pronoun may not occur when it is first encountered, it can occur at a later point if information is available to inform that process. This resolution happens even under shallow processing. With respect to unambiguous pronouns, if there is a possible referent available that matches the gender of the unambiguous pronoun then a link is made during co-indexation between pronoun and referent. This supports the position of Rigalleau et al. in that this co-indexation stage does not result in a processing cost.

In addition to the theoretical position we have outlined above, our findings also provide a strong methodological demonstration. They show that the type of comprehension question that readers have to answer can have an impact on the manner of processing. When readers know that they will have to answer questions testing how they interpreted the pronoun, they will want to be sure that they have arrived at a clear interpretation. The demand to answer the questions following the experimental sentences will cause readers to consider carefully the two alternative interpretations of the pronoun. Only when the alternatives have been examined will readers be 
able to make a strong commitment to one over the other. In our shallow-processing conditions, readers know that the questions they will be faced with can be answered equally well regardless of how they interpreted the pronoun. Therefore they will construct an underspecified representation that does not involve an initial co-indexation of an ambiguous pronoun. Interpretation only occurs when information is available to allow the correct assignment to be made. In line with other recent work it seems to be the case that readers construct underspecified representations associated with ambiguous pronouns when these are sufficient for the experimental task and when no disambiguating information is available. However, when disambiguating information does become available, this representation becomes more fully specified. This occurs even under shallow processing. Our data therefore also indicate that it is important for future research examining the time course of processing to carefully control the type of comprehension question that participants have to answer.

Original manuscript received 6 January 2006 Accepted revision received 29 November 2006 First published online 17 May 2007

\section{REFERENCES}

Arnold, J. E., Eisenband, J. G., Brown-Schmidt, S., \& Trueswell, J. C. (2000). The rapid use of gender information: Evidence of the time course of pronoun resolution from eyetracking. Cognition, 76, B13-B26.

Christianson, K., Hollingworth, A., Halliwell, J. F., \& Ferreira, F. (2001). Thematic roles assigned along the garden path linger. Cognitive Psychology, 42, 368-407.

Crawley, R. A., Stevenson, R. J., \& Kleinman, D. (1990). The use of heuristic strategies in the interpretation of pronouns. Journal of Psycholinguistic Research, 19, 245-264.

Ehrlich, K., \& Rayner, K. (1983). Pronoun assignment and semantic integration during reading-eye-movements and immediacy of processing. Journal of Verbal Learning and Verbal Behaviour, $22,75-87$.
Ferreira, F. (2003). The misinterpretation of noncanonical sentences. Cognitive Psychology, 47, 164-203.

Ferreira, F., Bailey, K. G. D., \& Ferraro, V. (2002). Good-enough representations in language comprehension. Current Directions in Psychological Science, 11, 11-15.

Garnham, A., Oakhill, J., \& Cruttenden, H. (1992). The role of implicit causality and gender cue on the interpretation of pronouns. Language and Cognitive Processes, 7, 231-255.

Gernsbacher, M. A., \& Hargreaves, D. J. (1988). Accessing sentence participants: The advantage of first mention. Journal of Memory and Language, 27, 699-717.

Greene, S., McKoon, G., \& Ratcliff, R. (1992). Pronoun resolution and discourse models. Journal of Experimental Psychology: Learning, Memory and Cognition, 18, 266-283.

Klin, C. M., Guzman, A. E., Weingartner, K. M., \& Ralano, A. S. (2006). When anaphor resolution fail: Partial encoding of anaphoric inferences. Journal of Memory and Language, 54, 131-143.

Klin, C. M., Weingartner, K. M., Guzman, A. E., \& Levine, W. H. (2004). Readers' sensitivity to linguistic cues in narratives: How salience influences anaphor resolution. Memory \& Cognition, 32, 511-522.

Levine, W. H., Guzman, A. E., \& Klin, C. M. (2000). When anaphor resolution fails. Journal of Memory and Language, 43, 594-617.

MacWhinney, B., St James, J., Schunn, C., Li, P., \& Schneider, W. (2001). STEP-a system for teaching experimental psychology using E-prime. Behavior Research Methods Instruments and Computers, 33, 287-296.

McDonald, J. L., \& MacWhinney, B. (1995). The timecourse of anaphor resolution-effects of implicit verb causality and gender. Journal of Memory and Language, 34, 543-566.

Rigalleau, F., \& Caplan, D. (2000). Effects of gender marking in pronominal coindexation. Quarterly Journal of Experimental Psychology, 53A, 23-52.

Rigalleau, F., Caplan, D., \& Baudiffier, V. (2004). New arguments in favour of an automatic gender pronominal process. Quarterly Journal of Experimental Psychology, 57A, 893-933.

Sanford, A. J., \& Garrod, S. C. (1989). What, when and how? Questions of immediacy in anaphoric reference resolution. Language and Cognitive Processes, 4, 235262. 
Sanford, A. J., \& Sturt, P. (2002). Depth of processing in language comprehension: Not noticing the evidence. Trends in Cognitive Sciences, 6, 382-386.

Stewart, A. J., Pickering, M. J., \& Sanford, A. J. (2000). The time course of the influence of implicit causality information: Focus versus integration accounts. Journal of Memory and Language, 42, 423-443.

Sturt, P. (2003). The time-course of the application of binding constraints in reference resolution. Journal of Memory and Language, 48, 542-562.
Van Gompel, R. P. G., Pickering, M. J., \& Traxler, M. J. (2000). Unrestricted race: A new model of syntactic ambiguity resolution. In A. Kennedy, R. Radach, D. Heller, \& J. Pynte (Eds.), Reading as a perceptual process (pp. 621-648). Oxford: Elsevier.

Van Gompel, R. P. G., Pickering, M. J., \& Traxler, M. J. (2001). Reanalysis in sentence processing: Evidence against current constraint-based and twostage models. Journal of Memory and Language, 45, $225-258$.

\section{APPENDIX}

\section{Materials}

The second sentence in each pair appeared in Experiment 2. The first question after each pair was used in the shallow-processing condition while the second question was used in the deep-processing condition.

1. Paul lent Rick/Kate the CD before she/he left for the holidays. She/he went to the Bahamas and sent Paul/Rick/Kate a postcard from the hotel.

Did Paul lend Rick the CD?

Who left for the holidays? Rick/Kate Paul

2. Claire laughed at Sue/Tom when she/he understood the joke. She/he usually had to ask Claire/Sue/Tom to explain the punchline.

Did Claire laugh at Sue?

Who understood the joke? Claire Sue/Tom

3. Fred scolded Brian/Mary after she/he finished playing the computer. She/he got the computer game from Fred/Brian/Mary.

No question.

Who finished playing the computer? Brian/Mary Fred

4. Craig/Cathy was talking to Neil about the football game seconds before she/he crashed the car. She/he was driving the car because Cathy/Neil/Cathy felt unwell.

No question.

Who crashed the car? Craig/Cathy Neil

5. Jessica telephoned Sarah/Mikey before she/he left for the airport. She/he missed the flight and called Jessica/Sarah/Mikey for help.

Did Sarah telephone Jessica?

Who left for the airport? Sarah/Mikey Jessica

6. Rebecca asked Sadie/Peter for a favour before she/he heard what was going on. She/he hadn't heard the news and Rebecca/ Sadie/Peter said nothing.

No question.

Who heard what was going on? Rebecca Sadie/Peter

7. James/Paula gave Sid the fiver as she/he walked towards the nightclub. She/he was excited because James/Sid/Paula knew the DJ. 
No question.

Who walked towards the nightclub? Sid James/Paula

8. Rachael whispered the answer to Anne/Fred as she/he had to leave early. She/he had an appointment and Rachael/Anne/Fred agreed to pass on the results.

Did Anne whisper the answer to Rachael?

Who had to leave early? Rachael Anne/Fred

9. Luke/Lucy pointed out a book to Will as she/he browsed the Amazon website. She/he was looking for Christmas presents and Luke/Will/Lucy had suggested Amazon.

No question.

Who browsed the Amazon website? Will Luke/Lucy

10. Jo emailed Lucy/Doug before she/he discovered that the rumours were true. She/he was happy after Jo/Lucy/Doug told her the rumours were exaggerated.

No question.

Who discovered that the rumours were true? Jo Lucy/Doug

11. Steve/Sarah told Jim about the problem just before she/he left the office. She/he was unhappy but said goodbye as Jim/Steve/ Sarah left.

Did Steve tell Jim about the problem?

Who left the office? Jim Steve/Sarah

12. Helena talked with Gemma/Steve as she/he walked to the train station. She/he said goodbye just as Helena/Gemma/Steve entered the station.

No question.

Who walked to the train station? Helena Gemma/Steve

13. Graham spoke to Sam/Jan about the job offer as she/he loaded the van. She/he placed the last box in the front as Sam/Graham/ Jan closed the back doors.

No question.

Who loaded the van? Sam/Jan Graham

14. Richard/Lindsay asked Carol for the money as she/he walked across the road. She/he made it across and went with Lindsay/ Carol/Richard to the bank.

Did Carole ask Lindsay for the money?

Who walked across the road? Lindsay/Richard Carol

15. Ron winked conspiratorially at Nick/Anne as she/he walked into the lobby. She/he stopped at the reception desk as Ron/Nick/ Anne walked out.

No question.

Who walked into the lobby? Nick/Anne Ron

16. Charlotte passed Nicola/Joseph the note as she/he walked into the boardroom. She/he took her place at the table as Charlotte/ Nicola/Joseph began the speech.

No question.

Who walked into the boardroom? Charlotte Nicola/Joseph

17. Catherine/Jonathon gave Hannah advice about the essay as she/he sat in the café. She/he rocked on the chair and drank coffee with Catherine/Hannah/Jonathon.

No question.

Who sat in the café? Catherine/Jonathon Hannah 


\section{STEWART, HOLLER, KIDD}

18. Matt/Mary returned the book to Harry just before she/he moved down to London. She/he had been looking forward to living there but realized he missed Matt/Harry/Mary quite a bit.

No question.

Who moved down to London? Harry Matt/Mary

19. Ian handed the racket to Thomas/Daphne as she/he waited in the sports club foyer. She/he had been waiting for ages but Ian/ Thomas/Daphne had just arrived.

Did Thomas hand the racket to Ian?

Who waited in the sports club foyer? Ian Thomas/Daphne

20. Laura/Derek handed the microphone to Caroline before she/he ran across the stage. She/he liked making a dramatic entrance and always waved at Laura/Carolina/Derek after the first song.

No question.

Who ran across the stage? Caroline Laura/Derek

21. Mike/Anna asked Dave about the fight when she/he arrived at work that morning. She/he was late but decided to talk to Mike/ Dave/Anna anyway.

No question.

Who arrived at work that morning? Mike/Anna Dave

22. Kim/Bob nodded at Liz as she/he walked into the courtroom. She/he took her seat as Kim/Liz/Bob questioned the witness.

Did Kim nod at Liz?

Who walked into the courtroom? Liz Kim/Bob

23. Martin whispered something to Oliver/Hilary before she/he started the lecture. She/he began talking into the microphone before realizing that Martin/Oliver/Hilary had broken the projector.

No question.

Who started the lecture? Martin Oliver/Hilary

24. Jane gave the script to Hayley/Simon as she he entered the TV studio. She/he had realized there was a page missing so asked Jane/Hayley/Simon for a new copy.

No question.

Who entered the TV studio? Hayley/Simon Jane 